\title{
Grey matter volume in healthy and epileptic beagles using voxel-based morphometry - a pilot study
}

\author{
Lisa Frank $^{1 *}$ D, Matthias Lüpke², Draginja Kostic ${ }^{1}$, Wolfgang Löscher ${ }^{3}$ and Andrea Tipold ${ }^{1}$
}

\begin{abstract}
Background: One of the most common chronic neurological disorders in dogs is idiopathic epilepsy (IE) diagnosed as epilepsy without structural changes in the brain. In the current study the hypothesis should be proven that subtle grey matter changes occur in epileptic dogs. Therefore, magnetic resonance (MR) images of one dog breed (Beagles) were used to obtain an approximately uniform brain shape. Local differences in grey matter volume (GMV) were compared between 5 healthy Beagles and 10 Beagles with spontaneously recurrent seizures ( 5 dogs with IE and 5 dogs with structural epilepsy (SE)), using voxel-based morphometry (VBM). T1W images of all dogs were prepared using Amira 6.3.0 for brain extraction, FSL 4.1.8 for registration and SPM12 for realignment. After creation of tissue probability maps of cerebrospinal fluid, grey and white matter from control images to segment all extracted brains, GM templates for each group were constructed to normalize brain images for parametric statistical analysis, which was achieved using SPM12.

Results: Epileptic Beagles (IE and SE Beagles) displayed statistically significant reduced GMV in olfactory bulb, cingulate gyrus, hippocampus and cortex, especially in temporal and occipital lobes. Beagles with IE showed statistically significant decreased GMV in olfactory bulb, cortex of parietal and temporal lobe, hippocampus and cingulate gyrus, Beagles with SE mild statistically significant GMV reduction in temporal lobe $(p<0.05$; family- wise error correction).

Conclusion: These results suggest that, as reported in epileptic humans, focal reduction in GMV also occurs in epileptic dogs. Furthermore, the current study shows that VBM analysis represents an excellent method to detect GMV differences of the brain between a healthy dog group and dogs with epileptic syndrome, when MR images of one breed are used.
\end{abstract}

Keywords: Voxel-based morphometry, Dogs, Epilepsy, Grey matter, MRI

\section{Background}

Dogs with epilepsy are frequent patients in veterinary practice [1]. Idiopathic epilepsy (IE) is one of the most common chronic neurological disorders in dogs, which is defined as a disorder of the brain characterized by spontaneous recurrent epileptic seizures of unknown, genetic or suspected genetic origin [1-3]. A genetic component was considered in several breeds [4], especially in Australian Shepherds [5], Beagles [6], Belgian Shepherds [7], Border Collies [8], English Springer

\footnotetext{
* Correspondence: Lisa.Frank@tiho-hannover.de

${ }^{1}$ Department of Small Animal Medicine and Surgery, University of Veterinary

Medicine, Hannover, Germany

Full list of author information is available at the end of the article
}

Spaniels [9], Golden Retrievers [10], Keeshonds [11], Labrador Retrievers [12], Lagotto Romagnolos [13, 14], Viszlas [15] and others. Affected dogs mostly have their first epileptic seizure with an age of 6 months to 6 years, interictal general and neurological examinations are normal $[16,17]$. Because IE is a diagnosis of exclusion, several diagnostic approaches have to be considered: TIER I: history, general and neurological examination, blood tests and urine analysis, TIER II: adding magnetic resonance imaging (MRI) and cerebrospinal fluid (CSF) analyses, TIER III: in addition electroencephalographic examinations [17]. Structural epilepsy (SE), caused by intracranial lesions like vascular damage, inflammation, trauma, anomalies or neoplasia of the brain, or reactive 
seizures are further reasons for the occurrence of seizures [1, 17]. For diagnostic purposes MRI of the brain is used to detect structural changes [1] resp. to diagnose IE by exclusion of such changes [18]. However, using new techniques such as volumetric studies in human epileptic patients, a reduced or increased volume of several grey matter (GM) structures could be detected using voxel- based morphometry (VBM) [19].

VBM is an automatically computational quantitative method to analyse MR images and detect differences in brain morphologies for instance in grey matter volume (GMV), white matter volume (WMV) or other structures between two groups of subjects [19]. After several pre-processing steps MR images are normalized to a consistent space for comparing each voxel of the same region in MR images of subjects by statistical analysis [19-21]. Resulting differences in brain volumes can be displayed in statistical parametric maps (SPM) [20]. Only few volumetric studies have been performed in veterinary medicine, since the diversity of head and brain shape between dog breeds [22] limit the application of VBM in dogs. In one volumetric study differences in the ratio of ventricular system volume to brain volume between dogs with IE and a healthy dog group were detected as preliminary results, while white matter (WM) volume to GMV ratio was not different between these groups [23]. In another study abnormalities of the hippocampus were seen in $12 \%$, an unilateral atrophy of hippocampus in $48 \%$ of dogs with IE compared to healthy dogs by measuring the surface areas of the hippocampus in each MR image plane [24]. No significant differences in hippocampus volume between dogs with IE and controls were found by Milne et al. [25]. Only after the reduction of the $95 \%$ reference interval in the chosen analysis an atrophy of hippocampus in 18 of 74 dogs with IE could be shown [25].

In the current study the VBM method was chosen because of its sensitivity in detecting subtle structural alterations, which cannot be measured by conventional MRI [26]. Aim of this study was to examine whether GMV differences exist between healthy and epileptic Beagles. This study was conducted in only one dog breed to examine an approximately uniform brain shape. The hypothesis should be proven that epileptic Beagles have reduced GMV, especially in hippocampus and basal nuclei, as well as increased GMV in frontal regions of the brain.

\section{Methods}

\section{Animals}

Fifteen Beagles were included in this study. Five Beagles (2 male, 3 male-neutered) with an average age of 29.6 months and an average weight of $17 \mathrm{~kg}$, property of the Department of Small Animal Medicine and Surgery of the University of Veterinary Medicine Hannover, represent the healthy control group (seizure-free up to 10 years of age). Their T1 weighted (T1W) MR images were already available from another animal experiment [27]. The patient group included 10 client-owned Beagles ( 2 female, 3 female-neutered, 3 male, 2 maleneutered) with an average age of 73.5 months and an average weight of $19.5 \mathrm{~kg}$, presented between 2012 to 2017 at the Department of Small Animal Medicine and Surgery of the University of Veterinary Medicine Hannover with a history of recurrent generalized tonicclonic seizures. No significant difference occurred between the age of controls and Beagles with IE $(p=0.07)$, however the age between controls and Beagles with SE differed significantly $(p=0.003)$. The weight was similar between controls and all epileptic Beagles $(p=0.30)$. All epileptic Beagles received comprehensive diagnostic workup based on the described TIER II level [17] on request of their owners. IE could be diagnosed in 5, structural changes (vascular, inflammatory, neoplasia) of the brain and thus SE in the remaining 5 Beagles (Table 1). In August 2017 all owners were called by phone to receive further information on seizure frequency resp. outcome of the disease. By telephone conference eight of ten owners could be reached.

\section{Magnetic resonance imaging (MRI)}

T1W three-dimensional (3D) turbo field echo (TFE) images of all fifteen Beagles were performed with a Phillips Achieva 3 Tesla MRI Scanner (Phillips Medical Systems, Eindhoven, The Netherlands) with a circular surface coil with a diameter of $11 \mathrm{~cm}$. Examinations were performed under general anesthesia and artificial ventilation. For premedication either acepromazine $(0.05 \mathrm{mg} / 10 \mathrm{~kg} \mathrm{BW}$ IM) or diazepam $(0.5 \mathrm{mg} / \mathrm{kg} \mathrm{BW} \mathrm{IV)} \mathrm{together} \mathrm{with}$ levomethadone $(0.2-0.6 \mathrm{mg} / \mathrm{kg}$ BW IV) were used. Anesthesia was induced with propofol ( $2 \mathrm{mg} / \mathrm{kg} \mathrm{BW} \mathrm{IV)}$ and maintained with isoflurane in air and oxygen. For image acquisition, dogs were placed in dorsal recumbency and the following protocol parameters were used: a repetition time of $11.2 \mathrm{~ms}$ to $11.5 \mathrm{~ms}$ with an echo time of $5.2 \mathrm{~ms}$ to $5.3 \mathrm{~ms}$, a slice thickness of $0.7 \mathrm{~mm}$ and a slice interval of $0.0 \mathrm{~mm}$. The flip angle was $8^{\circ}$ and the field of view (FOV) varied from $210 \mathrm{~mm}$ to $220 \mathrm{~mm}$.

\section{Pre-processing (Fig. 1)}

All fifteen T1W MR images were manually extracted using the software Amira 6.3.0 (FEI, part of Thermo Fisher Scientific, Hillsboro, Oregon, USA; costs: 5500US\$) to receive 3D brain images. With the FSL software version 4.1.8 (FMRIB Software Library, the University of Oxford, https:// fsl.fmrib.ox.ac.uk/fsl/fslwiki/FslInstallation; free download available) all fifteen extracted brain images were matched to the image of one control Beagle with a 12 parameter affine 
Table 1 Overview of Beagles and volume measurements of GMV, WMV and WBV achieved by using SPM12

\begin{tabular}{|c|c|c|c|c|c|c|c|}
\hline Beagle & Sex & Age (mo) & Weight (kg) & Group & GMV (ml) & WMV (ml) & WBV (ml) \\
\hline$\overline{C 1}$ & $m$ & 14 & 17.2 & Con & 45.2 & 31.7 & 76.9 \\
\hline$C 2$ & $\mathrm{~m}$ & 16 & 17 & Con & 44.9 & 36.0 & 80.9 \\
\hline C3 & $m n$ & 65 & 15.5 & Con & 43.1 & 36.0 & 79.1 \\
\hline $\mathrm{C} 4$ & $\mathrm{mn}$ & 38 & 15.2 & Con & 42.8 & 39.3 & 82.1 \\
\hline$C 5$ & $m n$ & 15 & 20.5 & Con & 45.9 & 35.7 & 81.6 \\
\hline P1 & $f$ & 109 & 13.2 & SE & 40.4 & 37.2 & 77.6 \\
\hline P2 & fn & 28 & 15.9 & IE & 47.7 & 36.0 & 83.7 \\
\hline P3 & $f$ & 82 & 24 & SE & 35.2 & 43.2 & 78.4 \\
\hline P4 & $m n$ & 45 & 19.7 & IE & 39.2 & 42.7 & 81.9 \\
\hline P5 & fn & 60 & 18 & SE & 48.7 & 34.2 & 82.9 \\
\hline P6 & fn & 104 & 14.8 & IE & 41.5 & 37.9 & 79.4 \\
\hline P7 & $\mathrm{m}$ & 53 & 15.2 & $\mathrm{IE}$ & 40.2 & 40.1 & 80.3 \\
\hline P8 & $m n$ & 82 & 26.8 & $\mathrm{IE}$ & 28.9 & 50.9 & 79.8 \\
\hline P9 & $\mathrm{m}$ & 96 & 23 & SE & 45.6 & 34.9 & 80.5 \\
\hline \multirow[t]{5}{*}{ P10 } & $\mathrm{m}$ & 76 & 25 & SE & 48.2 & 25.6 & 73.8 \\
\hline & & & & Average Con & 44.4 & 35.7 & 80.1 \\
\hline & & & & Average IE & 39.5 & 41.9 & 81.4 \\
\hline & & & & Average SE & 43.6 & 35.0 & 78.6 \\
\hline & & & & Average Epi & 41.6 & 38.5 & 80.1 \\
\hline
\end{tabular}

$C$ control, $P$ patient, $f$ female, $m$ male, $f n$ female-neutered, $m n$ male-neutered, mo months, $k g$ kilogram, Con controls, IE idiopathic epilepsy; $S E$ structural epilepsy, Epi epileptic, GMV grey matter volume, WMV white matter volume, WBV whole brain volume, $\mathrm{ml}$ milliliters

transformation kernel (Registration). Same software segmented the 5 extracted brain images of control Beagles in GM, WM and CSF (Segmentation). For this step, averaged intensity values of each tissue type in each brain image region read from a histogram produced by FSL 4.1.8 and displaying frequency of every single intensity value in a brain image region [28] were applied. To reach an automatical segmentation, tissue probability maps (TPMs) of GM, WM and CSF were created. Therefore all 5 extracted GM, WM and CSF images of control Beagles were realigned to each other (Realignment) and a template for each tissue type was generated by Dartel-Toolbox in the software SPM12 (Wellcome Department of Cognitive Neurology, London, UK, http://www.fil.ion.ucl.ac.uk/spm/ software; free download available) that was implemented in MATLAB 9.2 R2017a (The MathWorks, Natick, Massachusetts, USA; costs: $500 € /$ year). Templates were smoothed with a $2 \mathrm{~mm}$ FWHM (Full Width Half Maximum) Gaussian kernel (Smoothing). Resulting TPMs were used to segment all 15 extracted brain images automatically with SPM12 (Segmentation). By re-using DartelToolbox in SPM12, 4 GM templates were created representing an average of extracted GM images: extracted GM images of the 5 control Beagles produced the standard template, further, 3 study specific templates emerged: one of the 5 extracted GM images from Beagles with IE, one of the 5 extracted GM images from Beagles with SE and one of all 10 extracted GM images from all epileptic Beagles.

To guarantee a voxel-wise comparison of GMV between groups, each extracted GM image was spatially normalized to an uniform space using flow fields by SPM12 (Normalization). These flow fields contain information of the necessary deformations by template creation and were created in this step. While normalization, smoothing of extracted GM images with a $2 \mathrm{~mm}$ FWHM Gaussian kernel was applied again as described by others [29].

\section{Statistical analysis using voxel-based morphometry}

After the described steps extracted, registered, segmented, realigned, smoothed and spatially normalized GM images were taken for statistical analysis by using SPM12 [28-30]. Three VBM analyses were performed and a two-sample t-Test was chosen to compare GMV differences between groups. In analysis 1 control Beagles group were compared with IE Beagles group, in analysis 2 control Beagles group with SE Beagles group and in analysis 3 control Beagles group with epileptic Beagles group (IE and SE Beagles). Whole brain volume (sum of GMV and WMV) of each brain was taken as a covariant to correct for some large brain differences between study Beagles. Computation of brain volume (Table 1) was achieved by using the toolbox tissue volume in SPM12. 



$\bigcup$ Statistical analysis

Fig. 1 Overview of VBM pre-processing steps: 1: original T1W images; 2: extracted brain images; 3: registered brain images; 4: WM, GM, CSF images segmented by the software FSL; 5: realigned WM, GM, CSF images; 6: templates of WM, GM, CSF; 7: TPMs of WM, GM, CSF; 8: WM, GM, CSF images segmented by the Software SPM using TPMs; 9: template of control, IE, SE and epileptic Beagles; 10: flow fields; 11: normalized GM images (VBM: voxel- based Morphometry, T1W: T1 weighted, WM: white matter, GM: grey matter, CSF: cerebrospinal fluid, TPMs: tissue probability maps, IE: idiopathic epilepsy, SE: structural epilepsy)

In the option Masking, that pretends which voxel is included in the analysis [31], every voxel with a value of 0 (implicit masking) or a value below 0.01 (threshold masking with an absolute threshold of 0.01) was excluded from the analysis. The rest of settings in this step were adopted as required. The classic method was set in the Estimate option, in which the analysis is passed through. To show the results of $\mathrm{t}$-statistics, two $t$-contrasts were 
defined: contrast a (1-1) defined that control Beagles group has larger contrast values than IE/SE/epileptic Beagles group, contrast b $(-11)$ defined that control Beagles group has less contrast values than IE/SE/epileptic Beagles group [30]. Furthermore, a FWE (family- wise error correction) for controlling chance of false positives [21] with a $p$-value $<0.05$ was used as well as a minimum limit for displaying clusters with at least 3 resp. 10 voxels (extend threshold set to 3 resp. 10). Extend threshold of 3 voxels was chosen in order to minimize the occurrence of isolated randomly changed voxels in illustration of the VBM results, 10 voxels to represent larger GMV differences between groups that might have a clinical relevance. Therefore, all voxels of the GM that differed between groups with significance to a $p$ value of $<0.05$ and joined together in clusters with at least 3 resp. 10 voxels were shown as statistical parametric maps for instance in color maps [21].

\section{Results}

The results of VBM statistics were displayed in statistical parametric maps in different views to identify the exact structures of GMV differing between groups of subjects. Specification of anatomical structures is based on definitions published by Uemura [32], Stoffel [33] and Palazzi [34].

\section{Group comparison}

A comparison of the following groups using VBM was performed: controls and Beagles with IE, controls and Beagles with SE, controls and all epileptic Beagles, Beagles with IE and SE.

\section{Controls vs. IE}

When compared to controls using a threshold of FWEcorrected $p<0.05$ and an extend threshold of 3 voxels, IE Beagles had statistically significant GMV reductions in the olfactory bulb bilaterally as well as in both cingulate gyri. Furtheron, statistically significant decreased GMV was found in the cortex of parietal (GM below coronal sulcus bilaterally and right marginal sulcus) and temporal lobe (left sylvian gyrus) and the hippocampus bilaterally (Table 2, Figs. 2 and 3). VBM comparison of IE group with control group also yielded statistically significant increased GMV in IE Beagles in the cortex of frontal (rostral part of right suprasylvian gyrus) and parietal lobe (precruciate gyrus near or in cruciate sulcus) as well as in the left piriform lobe (Table 3, Fig. 3). Setting the extend threshold to 10 voxels to show eventually clinical relevant aspects still displayed significant decreased GMV in IE Beagles in left olfactory bulb, left cingulate gyrus and left hippocampus compared to healthy Beagles (Table 2). Increased GMV regions did not reach this threshold.

\section{Controls vs. SE}

In Beagles with SE statistically significant decreased GMV could be seen in the cortex of the temporal lobe (GM below the left medial ectosylvian sulcus) and occipital lobe in comparison to controls with FWEcorrected $p$-value $<0.05$ and extend threshold of 3 voxels (Table 2). In contrast the GMV was statistically significant increased in the cortex of parietal lobe (region beneath the middle part of the left coronal sulcus) (Table 3). Setting the extend threshold to 10 voxels for clinical relevant GMV changes displayed no significant differences in GMV between control and SE Beagles.

\section{Controls vs. epileptic}

Comparing controls with all 10 epileptic Beagles $(p<0.05$ FWE, extend threshold 3) several statistically significant changes of GMV in epileptic Beagles were detected. Regions with statistically significant decreased GMV were found in the olfactory bulb bilaterally, the cortex of frontal, parietal and temporal lobe. Further on, GMV reduction in epileptic Beagles was significant in both cingulate gyri and right and left putamen and claustrum $(p<0.05)$ (Table 2, Fig. 4). Epileptic Beagles also had decreased GMV in the hippocampus bilaterally, spacious regions of the occipital lobe and in cerebellum bilaterally in comparison to healthy Beagles (Table 2, Fig. 4). Statistically significant enhanced GMV in epileptic Beagles was found in the laterocaudal area of the right olfactory bulb, the cortex of temporal lobe and piriform lobe bilaterally (Table 3 ). Changing of extend threshold to 10 voxels emerged statistically significant reduced GMV in epileptic Beagles compared to controls in olfactory bulb, some parts of the cortex of frontal, parietal, temporal and occipital lobes, cingulate gyrus, putamen, claustrum and both hippocampi as before and mildly statistically significant decreased GMV in cerebellum on right side (Table 2). At this extend threshold of 10 voxels, epileptic group compared to control group displayed statistically significant increased GMV in temporal lobe (Table 3).

\section{IE vs. SE}

No significant differences in GMV were detected between Beagles with IE and SE.

\section{Volume measurement and follow-up information by the owners}

Six of ten epileptic Beagles showed reduced, four of ten increased whole GMV in comparison to controls (Table 1). Eight of ten owners were reached to evaluate the epileptic Beagles current condition and the outcome of the disease. Five of six Beagles with decreased whole GMV did not have a favorable response to antiepileptic 
Table 2 Regions of statistically significant reduced GMV in IE, SE and epileptic Beagles compared to healthy control Beagles

\begin{tabular}{|c|c|c|c|c|c|}
\hline Major brain region & Region of reduced GMV & Voxel per cluster & Z score & PFWE-corr & Group \\
\hline \multirow[t]{2}{*}{ Olfactory } & left olfactory bulb & 34 & 5.54 & 0.000 & $\mathrm{IE}$ \\
\hline & right olfactory bulb & $4 / 3$ & $4.95 / 4.7$ & $0.011 / 0.02$ & \\
\hline \multirow[t]{6}{*}{ Parietal } & left cingulate gyrus & $\underline{15}$ & $\underline{5.49}$ & $\underline{0.001}$ & \\
\hline & right cingulate gyrus & 8 & 5.29 & 0.002 & \\
\hline & middle part cingulate gyrus & 6 & 5.22 & 0.003 & \\
\hline & GM below left coronal sulcus & 9 & 5.38 & 0.001 & \\
\hline & GM below right coronal sulcus & 3 & 5.00 & 0.009 & \\
\hline & GM below right marginal sulcus & 3 & 4.78 & 0.019 & \\
\hline \multirow[t]{3}{*}{ Temporal } & left sylvian gyrus & 6 & 4.87 & 0.017 & \\
\hline & left hippocampus & $\underline{10}$ & $\underline{5.23}$ & $\underline{0.003}$ & \\
\hline & right hippocampus & 7 & 4.91 & 0.014 & \\
\hline Temporal & GM below left medial ectosylvian sulcus & 3 & 5.03 & 0.008 & SE \\
\hline Occipital & left occipital gyrus & 3 & 4.88 & 0.017 & \\
\hline \multirow[t]{2}{*}{ Olfactory } & left olfactory bulb & $\underline{45}$ & $\underline{6.26}$ & $\underline{0.000}$ & Epileptic \\
\hline & $\underline{\text { right olfactory bulb }}$ & $\underline{51}$ & $\underline{6.19}$ & $\underline{0.000}$ & \\
\hline \multirow[t]{2}{*}{ Frontal } & $\underline{\text { frontal gyrus concentral }}$ & $\underline{31}$ & $\underline{5.90}$ & $\underline{0.000}$ & \\
\hline & right frontal gyrus & $\underline{10}$ & $\underline{5.70}$ & $\underline{0.000}$ & \\
\hline \multirow[t]{5}{*}{ Parietal } & left cingulate gyrus & $\frac{11 /}{11}$ & $\underline{5.81 / 5.47}$ & $\underline{0.000 / 0.001}$ & \\
\hline & $\underline{\text { right cingulate gyrus }}$ & $\begin{array}{l}5 / \\
14 \\
\end{array}$ & $5.16 / \underline{5.54}$ & $0.004 / \underline{0.000}$ & \\
\hline & central cingulate gyrus & $\underline{11}$ & $\underline{5.60}$ & $\underline{0.000}$ & \\
\hline & GM below left coronal sulcus & $\underline{73}$ & $\underline{5.97}$ & 0.000 & \\
\hline & $\underline{\text { GM below right coronal sulcus }}$ & $\underline{21}$ & $\underline{5.75}$ & $\underline{0.000}$ & \\
\hline \multirow[t]{13}{*}{ Temporal } & left putamen/claustrum & $\underline{35}$ & $\underline{5.69}$ & $\underline{0.000}$ & \\
\hline & $\underline{\text { right putamen/claustrum }}$ & $\underline{43}$ & $\underline{5.59}$ & $\underline{0.000}$ & \\
\hline & left sylvian gyrus & $\underline{44 / 8}$ & $\underline{6.42 / 5.13}$ & $\underline{0.000 / 0.004}$ & \\
\hline & right sylvian gyrus & 7 & 5.08 & 0.006 & \\
\hline & GM below left ectosylvian sulcus & $\underline{53}$ & $\underline{6.21}$ & $\underline{0.000}$ & \\
\hline & GM below right ectosylvian sulcus & $\underline{35}$ & $\underline{5.99}$ & $\underline{0.000}$ & \\
\hline & GM below left medial suprasylvian gyrus & $3 / 3$ & $4.81 / 4.72$ & $0.012 / 0.012$ & \\
\hline & GM below right medial suprasylvian gyrus & 4 & 4.94 & 0.012 & \\
\hline & right suprasylvian gyrus & 4 & 4.94 & 0.012 & \\
\hline & left hippocampus & $\underline{67 / 13 / 6}$ & $\underline{5.76 / 5.99 / 5.32}$ & $\underline{0.000 / 0.000 / 0.002}$ & \\
\hline & $\underline{\text { right hippocampus }}$ & $\underline{49 / 5 / 16}$ & $\underline{5.89 / 4.35 / \underline{6.26}}$ & $\underline{0.000 / 0.001 / \underline{0.000}}$ & \\
\hline & GM below left marginal sulcus & 4 & 5.38 & 0.001 & \\
\hline & $\underline{\text { GM below right marginal sulcus }}$ & $\underline{23}$ & $\underline{6.17}$ & 0.000 & \\
\hline \multirow[t]{2}{*}{ Occipital } & $\underline{\text { occipital gyrus }}$ & $8 / \underline{17 / 6 / 144}$ & $5.15 / \underline{5.39 / 5.21 / 6.28}$ & $0.004 / \underline{0.001 / 0.003 / 0.000}$ & \\
\hline & GM below medial suprasylvian gyrus & $3 / 3$ & $5.01 / 5.00$ & $0.008 / 0.009$ & \\
\hline \multirow[t]{2}{*}{ Cerebellar } & left cerebellum & $6 / 6 / 6 / 7$ & $5.85 / 5.53 / 5.37 / 5.09$ & $0.000 / 0.000 / 0.001 / 0.005$ & \\
\hline & right cerebellum & $9 / 12 / 7 / 5$ & $5.73 / 5.39 / 5.29 / 5.12$ & $0.000 / 0.001 / 0.002 / 0.005$ & \\
\hline
\end{tabular}



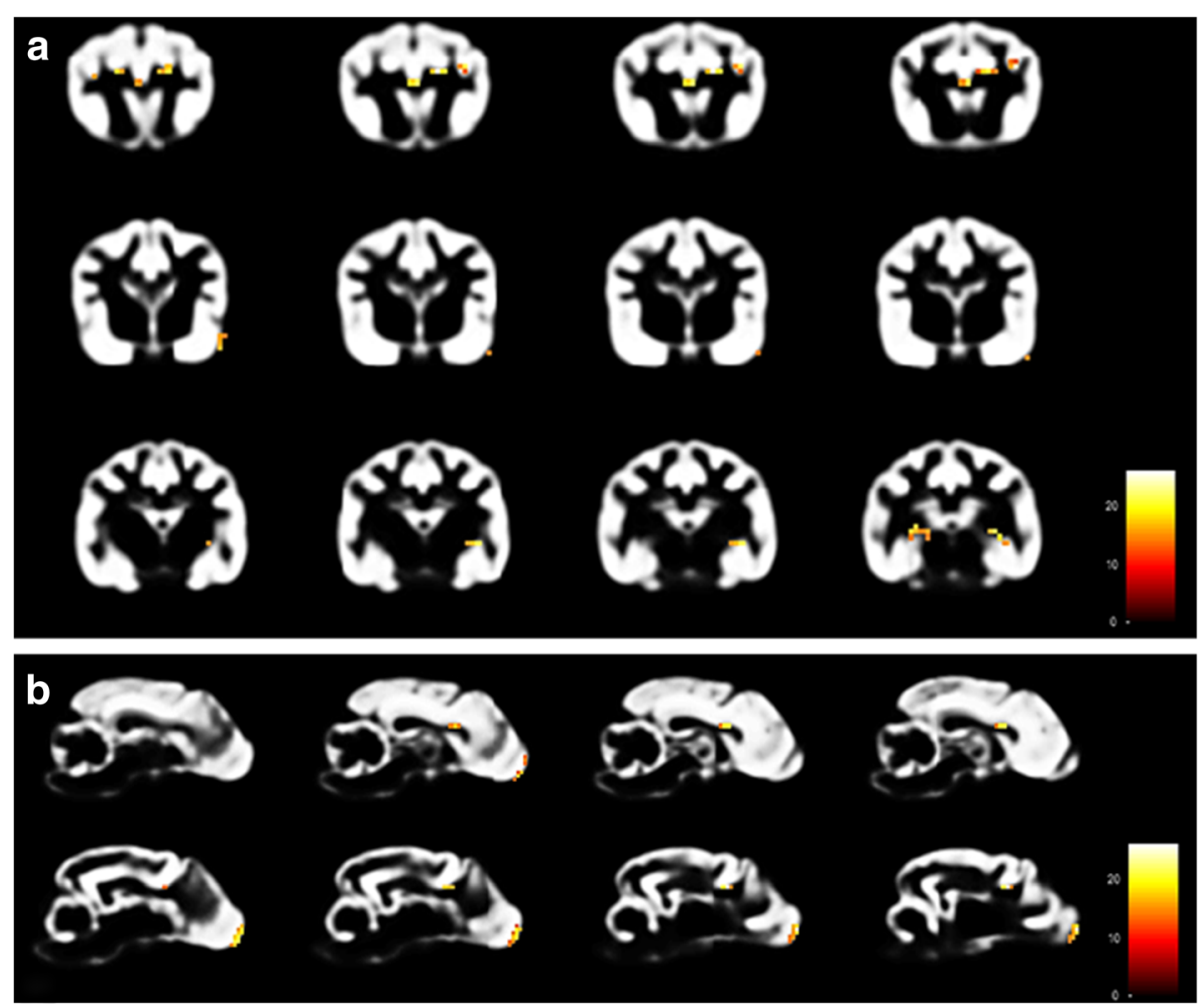

Fig. 2 Results of VBM analysis 1 (control vs. IE Beagle group) using SPM12. Transversal [a] and Sagittal [b] view of parts of the GM-template of control Beagles illustrating regions of significant reduced GMV (coloured voxels) in the olfactory bulb, cingulate gyrus, cortex of parietal and temporal lobe and hippocampus in the IE Beagle group (VBM: voxel-based morphometry, IE: idiopathic epilepsy, GM: grey matter, GMV: grey matter volume)

drugs (AED) treatment; indeed, three were euthanized because of status epilepticus resp. no response to AED treatment on request of their owners. Two dogs with regular seizure activity despite treatment are still alive. Only one of six Beagles with reduced whole GMV became seizure free, but was euthanized because of another disease than epilepsy. In contrast, two of two Beagles with increased whole GMV are alive and seizure-free under appropriate medication.

\section{Discussion}

Current pilot study was designed to examine grey matter volume differences between healthy control and epileptic dogs and to prove the feasibility of VBM methods in dogs. Results identified several brain regions with statistically significant reduced or increased GMV in Beagles with IE and SE compared to healthy control Beagles that are similar to in people. Most important significant volumetric differences were found in hippocampus, putamen/claustrum and cerebral cortex representing either the cause or consequence of epileptic seizures. Furthermore, in cingulate gyrus and frontal brain regions significant differences were detected that might explain behavioral changes in epileptic dogs.
VBM was chosen because of its sensitivity in detecting GMV differences between groups of healthy and epileptic humans, which cannot be seen in conventional MRI $[19,26]$. Used MR images should have a relatively high resolution and be made with an at least 1 or 1.5 Tesla MRI Scanner to avoid preprocessing errors like inaccurate extraction or segmentation [20].

Using the VBM method not only specific structures of the brain can be evaluated, but also anatomical differences of the entire brain [19, 20]. Furthermore, VBM allows the assessment of atrophic brain regions across large groups of subjects without manual measurements and subjective influences [21]. However, the application of VBM method for animals, especially for dogs, has some disadvantages. Specialists for performing and evaluating VBM are necessary and the access of high performance computers is limited in veterinary medicine [22]. Variety of existing dog breeds resulting in brachycephalic, mesocephalic, dolichocephalic and mixed skull and brain shapes complicates standardized image processing and using VBM methods in canines routinely $[22,35]$. In humans, skull shape with only minor variation in skull or brain size facilitates the application of VBM method. Customized brain templates, as they exist 


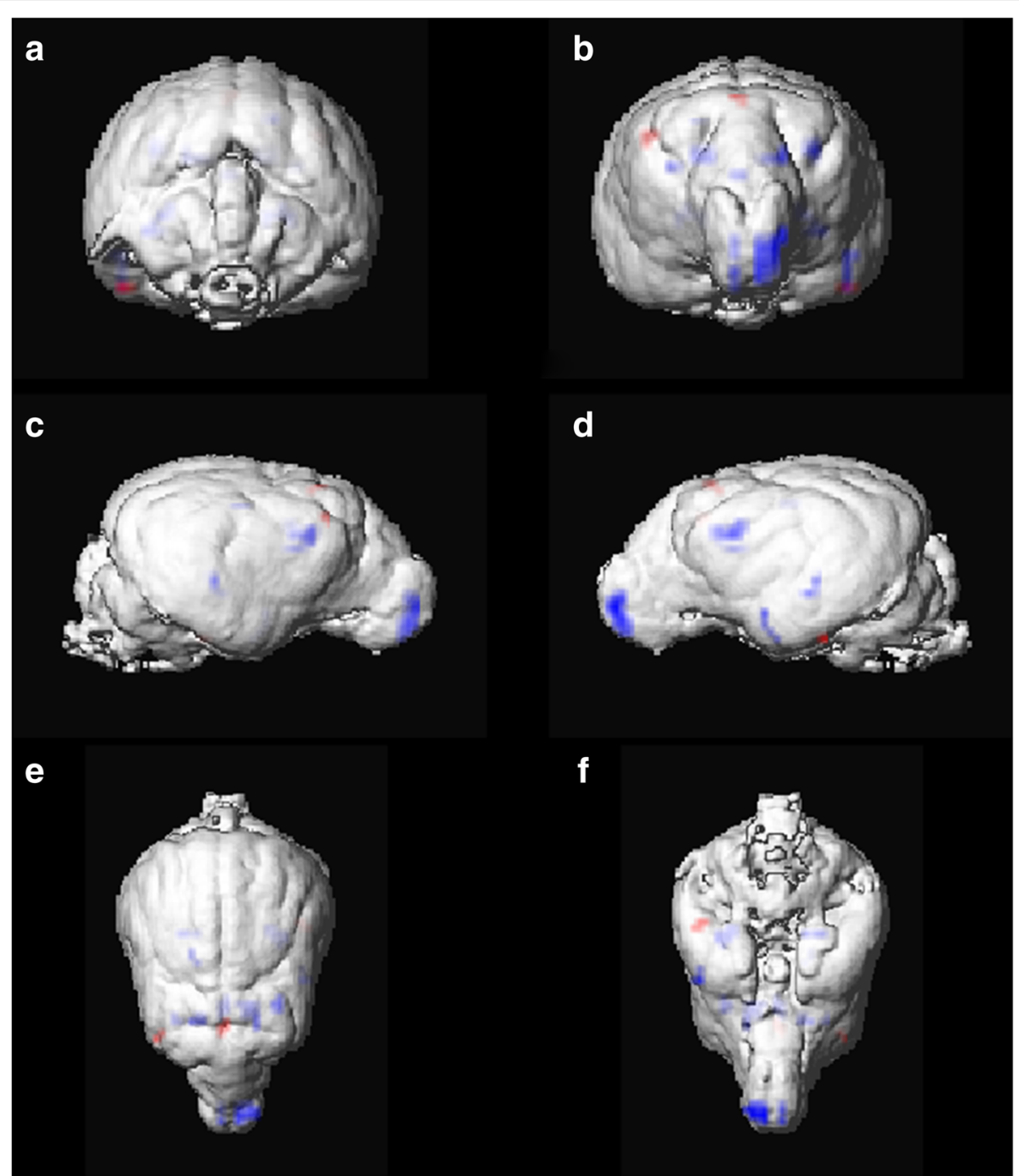

Fig. 3 Results of VBM analysis 1 (control vs. IE Beagle group) using SPM12 illustrated on surface rendering of mean extracted GM images of control Beagles. Statistically significant reduced (blue) and increased (red) GMV is displayed in caudal [a], rostral [b], lateral right [c] and left side [d], dorsal [e] and ventral [f] views (VBM: voxel-based morphometry, IE: idiopathic epilepsy, GM: grey matter, GMV: grey matter volume)

in human medicine, are rare or missing in veterinary medicine [22, 35]. Datta and others constructed a mesocephalic atlas of 15 brains of mesocephalic mixed-breed dogs [36], in another study a brachycephalic, mesocephalic and dolichocephalic as well as a mixed meso- and dolichocephalic skull shape specific template were created [22]. Two templates consisting of one dog breed, the Beagle [29], which is not publicly accessible, and the Dobermann Pinscher exist [37]. Such templates cannot be used for all dog breeds suffering from epilepsy. To overcome these difficulties, in the current study brain MR images of only one breed, the Beagle, were used to create a specific template for comparison analysis between healthy and epileptic brain MR images and to avoid analytical errors due to different brain shapes.
Application of VBM is widely used in human medicine in several neurological disorders [38-41], especially in epilepsy [42]. These studies detected subtle changes in brain volumes in epileptic humans [19, 26]. In contrast, VBM studies identifying structural brain changes in veterinary medicine are rare. Few studies have been performed in dogs to determine either brain volume in older dogs compared to younger ones [29], studying the influence of an antioxidant diet on cortical atrophy in aging dog brains [43] or structural brain abnormalities in canine compulsive disorders [37]. Other authors examined brain tissue volumes of dogs using volumetry [35] or studied the ventricular system to brain tissue volume ratio and WMV to GMV ratio, respectively [23], or hippocampal volume [44] between groups of healthy and epileptic dogs. 
Table 3 Regions of statistically significant increased GMV in IE, SE and epileptic Beagles compared to healthy control Beagles

\begin{tabular}{|c|c|c|c|c|c|}
\hline Major brain region & Region of increased GMV & Voxel per cluster & Z score & PFWE-corr & Group \\
\hline Frontal & right suprasylvian gyrus & 3 & 5.05 & 0.007 & IE \\
\hline Parietal & precruciate gyrus/cruciate sulcus & 4 & 5.11 & 0.005 & \\
\hline Temporal & left piriform lobe & 4 & 5.04 & 0.007 & \\
\hline Parietal & region coronal sulcus & 8 & 4.99 & 0.009 & SE \\
\hline Olfactory & right olfactory bulb & 3 & 4.90 & 0.015 & Epileptic \\
\hline \multirow[t]{6}{*}{ Temporal } & left sylvian gyrus & 14 & $\underline{5.28}$ & $\underline{0.002}$ & \\
\hline & right sylvian gyrus & 8 & 5.00 & 0.009 & \\
\hline & left suprasylvian gyrus cranial & $\underline{55}$ & $\underline{5.67}$ & $\underline{0.000}$ & \\
\hline & $\underline{\text { right suprasylvian gyrus cranial }}$ & $\underline{19 / 5}$ & $\underline{5.39 / 4.99}$ & $\underline{0.001 / 0.009}$ & \\
\hline & left piriform lobe & $\underline{42}$ & $\underline{5.92}$ & $\underline{0.000}$ & \\
\hline & right piriform lobe & 11 & $\underline{5.76}$ & 0.000 & \\
\hline
\end{tabular}

Underlined: significant changes in GMV even with an extend threshold of 10 voxels (GMV: grey matter volume, IE: idiopathic epilepsy, SE: structural epilepsy)

In the current study epileptic Beagles were examined. Described MR images of Beagle brains had to be manually extracted due to lack of a software that automatically extracts dog brains as accurately as manual extraction. Milne and others showed that manual brain extraction followed by the application of brain shape specific templates is the most accurate method for atlasbased segmentation in dogs [22]. Therefore, breed brain shape specific templates of GM, WM and CSF for a fully automatically brain segmentation was created [30, 31].
Smoothing with a $2 \mathrm{~mm}$ FWHM Gaussian kernel in the preprocessing step was chosen as described by others [29]. In order to minimize the occurrence of isolated randomly changed voxels in illustration of the VBM results the extend threshold of 3 voxels was chosen. In a second step only clusters with at least 10 altered voxels were displayed to represent large GMV differences between groups that might have clinical relevance.

Although VBM can detect differences in brain morphometry in smaller groups of subjects with expected large


Fig. 4 Results of VBM analysis 3 (control vs. epileptic Beagle group) using SPM12. Transversal [a] and Sagittal [b] view of parts of the GM-template of control Beagles illustrating regions of significant reduced GMV (coloured voxels) in the olfactory bulb, cortex of frontal, parietal, temporal and occipital lobe, cingulate gyrus, putamen, claustrum, hippocampus and cerebellum in the epileptic Beagle group (VBM: voxel-based morphometry, GM: grey matter, GMV: grey matter volume) 
volume differences, greater power is achieved in groups with a high number of participants $[20,21,45]$. In the current pilot study MR images of 5 IE as well as $5 \mathrm{SE}$ Beagles were used to evaluate the feasibility of the method for future studies with higher numbers of participants. Indeed, summarizing all 10 epileptic Beagles with spontaneous recurrent seizures, more frequent and larger GMV changes in statistical analysis were found in comparison to the evaluation of each group alone. Hence, VBM is a good method to detect brain volume changes between groups of subjects, but it cannot show reliable information for single-subject comparison [21]. VBM seems to be suitable for further epilepsy research in veterinary medicine evaluating certain groups of dogs with specific epileptic syndromes, but cannot improve the diagnostic workup of single epileptic patients.

Current VBM study revealed statistically significant reduced GMV in the frontal gyrus in epileptic dogs which is consistent with several previous studies made in human medicine. Reduced GMV was seen in some parts of the frontal lobe in patients with temporal lobe epilepsy (TLE) [46-50], juvenile myoclonic epilepsy (JME) [51], juvenile absence epilepsy (JAE) [52] and generalized tonic-clonic seizures (GTCS) [53] as well as frontal lobe epilepsy (FLE) [54]. Furthermore, statistically significant reduced GMV in several areas of the cortex of temporal and a few regions of parietal lobe in epileptic and IE Beagles and mildly statistically significant reduced GMV in the cortex of temporal lobe in SE Beagles was detected in the current study. Reduced GMV in cortices of temporal and parietal lobes are known in humans with TLE $[48,55]$. Decreased GMV in areas of temporal lobe in TLE patients $[45,46,56]$, in FLE patients [54] and patients with JAE [52] were also displayed in humans, while others found decreased GMV in parietal regions in brains of JME [57] or TLE patients [47, 49, 58]. Our study also revealed a statistically significant loss of GMV in occipital gyrus in SE and epileptic Beagles. These findings are consistent with several human medicine studies of TLE patients [46, 47, 49, 55, 58].

Many explanations for reduced GMV of the cerebral cortex in epileptic patients exist. Focal onset of epileptic seizures often spreads to the frontal lobe $[59,60]$ representing a pathway for interhemispheric propagation of ictal activity in humans with TLE [47]. In a family of Shetland Sheepdogs with IE Morita and others revealed an epileptic focus in the frontal lobe, which was consistent with acute neuronal necrosis [61]. Lateral TLE in humans seems to have a lateral temporal seizure onset [62]. Others hypothesize that hypometabolism in the temporal pole of humans with TLE may be responsible for GMV loss in this area [56]. Reduced GMV in temporal lobe cortex could even be involved in cognitive impairment in children with FLE [54]. In addition, the network of thalamus and the cerebral cortex might play a role in the mechanism of generalized seizures [42]. Reduced GMV in the cortex of frontal, parietal, temporal and occipital lobes in epileptic dogs might represent the onset focus of epileptic seizures, a lesion caused by epileptic seizures themselves or might be the consequence of hypometabolism in the affected regions. Behavioral changes occasionally observed in epileptic dogs [63] could also be the sequelae of decreased GMV in frontal parts of the brain as suggested by Woermann and colleagues in humans with TLE [58].

In contrast, increased GMV of frontal lobe areas was found in humans with JME [51, 57, 64, 65], TLE [47, 48], epileptic patients with hippocampal sclerosis [50] as well as FLE [54]. Keller and colleagues described additionally larger GMV in parietal cortex of human patients with TLE [47]. Even in epileptic humans with hippocampal sclerosis and TLE areas of increased GMV in temporal lobe could be detected [50, 58]. These results are consistent with findings of the current study. Mildly statistically significant increased GMV in the frontal lobe occurred in Beagles with IE compared to healthy controls as well as increased GMV in the cortex of parietal lobe in IE and SE Beagles. Furthermore, temporal lobe showed GM areas of statistically significant increased volume in epileptic Beagles. An increased grey matter volume could represent microdysgenesis and increased neuronal density [65-67] or reflect compensatory mechanisms such as increase in synapses because of reduced functional input to these areas [54]. Others suggest that higher frontal GMV in TLE patients before and after surgery with good outcome may reflect tissue recovery or protective mechanism for prevention of recurrent seizures [48]. A similar theory is that the cortex may play a leading role in seizure generation [57]. Higher GMV in cortex in epileptic Beagles may reflect tissue recovery or a compensatory mechanism because of reduced functional input in such brain areas. Furthermore, the cortex may play a leading role in seizure generation. Diminished GM to WM demarcation in temporal lobe as it is described in one human study in TLE patients [68] could be another explanation for our findings in epileptic Beagles.

Statistically significant reduced GMV in cingulate gyrus could be found in our study in epileptic dogs which is in accordance with several findings in human TLE $[45,46,69]$ as well as in epileptic humans with JME and juvenile $\mathrm{AE}[52,70]$. On the other hand, in one study increased GMV in this part of the brain was described in patients with TLE compared to healthy controls [47]. In humans, it is known, that in mesial temporal lobe epilepsy seizures propagate to frontal lobe but also to cingulate areas [46]. This might explain GMV decrease in cingulate gyrus in described Beagles as 
a consequence of seizures themselves. In contrast, cingulate gyrus could also be considered as seizure focus as described in idiopathic generalized epilepsy (IGE) in humans [71]. Since cingulate gyrus plays a role in processing emotions [72], atrophic changes might explain postictal or general behavioral changes in epileptic dogs.

Lower GMV in putamen detected in the described Beagles was also shown in VBM analyses of human patients with neocortical seizures [73], IGE [74, 75] and TLE $[45,76]$. Human patients with frontal, lateral temporal or occipital lobe seizures display a reduction in subcortical GMV including the putamen [73]. Because of connections between putamen and cerebral cortex [32], an abnormal cortex may result in fewer and aberrant dendrites projecting to the putamen and leading to GMV loss [75]. Since in the current study GMV loss was detected in cerebral cortex as well as in putamen, projection of dendrites damage is also feasible for epileptic Beagles. Furthermore, putamen atrophy may explain clinical symptoms like the tonic-clonic phase of generalized seizures [75].

The hippocampus of Beagles with IE had statistically significant reduced GMV that was even obvious, when all epileptic Beagles were calculated together. This finding is consistent with several human VBM studies about TLE $[45,46,49,55,56,69,77]$. Volumetric studies in veterinary medicine also showed hippocampal atrophy in epileptic dogs [24, 44]. Hippocampal sclerosis is the most common pathologic finding in human patients with mesial TLE $[45,78]$ and is suggested to be epileptogenic in patients with epilepsy [79]. Moreover, GMV loss in medial temporal lobe like hippocampus may also play a role in FLE and the etiology of cognitive impairment in epileptic patients [54]. Others suggest that damage of hippocampal neurons can also occur when the initializing seizure focus is located elsewhere [80]. Specific breed effects on hippocampal volume [24] and smaller hippocampal volumes in dogs of at least 11,5 years [81] were detected. Since the current study was performed in only one breed and the oldest epileptic Beagle was 9 years old, such physiological changes could be excluded and volume changes might be associated with epilepsy. Hippocampal atrophy is described as a consequence of seizures or status epilepticus in rats, mice and dogs [82-84] supporting the aforementioned assumption.

Current study even revealed statistically significant decreased GMV in cerebellum bilaterally. These findings are in accordance with results of several VBM studies of humans with TLE [45, 48, 49, 55, 69, 85], JME [51, 57] as well as GTCS [53]. The cerebellum has connections with a large number of other brain regions, especially the cerebral cortex $[51,86,87]$ leading to the hypothesis that nerve cell injury and discharges spread to cerebellum and cause Purkinje cell loss [87-89]. Moreover, cerebellar hypometabolism is detected in patients with focal seizures [90]. Whereas the cerebellum seems to have an inhibitory effect on seizures, cerebellar lesions could lead to generation and spread of epileptic seizures [87, 90].

In epileptic Beagles GM volume changes of the olfactory bulb were seen, but not further considered. Different sizes and shapes of each individual olfactory bulb were noticed during manual extraction of MR images and might represent normal variations of the brain.

Additionally, GM and WM volumes of all 15 Beagle brains were determined using tissue volume tool in SPM12 and compared with volumetric investigations by others [23, 35]. WMV of epileptic Beagles was higher than volumes measured by Schulze et al. [35]. Since control Beagles had similar WMV as the different breeds used in the other volumetric study [35], an increased WMV in Beagles with IE could be the consequence of the described partly reduction of GMV. This hypothesis is supported by changed GMV to WMV as well as GMV to WBV ratios in IE and epileptic Beagles of the current study in comparison to these ratios computed by Schulze and colleagues [35], while it contradicts the assumption that dogs with IE have reduced WBV with preservation of WMV to GMV ratio [23].

In the current study five of six epileptic Beagles with reduced whole GMV than controls showed an unfavorable outcome because of their unresponsiveness to antiepileptic therapy, whereas two of two Beagles with increased whole GMV are currently seizure-free under medication. These preliminary findings are supported by results of a human study that showed that TLE patients with significantly reduced GMV in the frontal lobe, presurgery, will more likely experience a poor seizure outcome, post-surgery, while patients with higher GMV in frontal lobe pre-surgery, experience seizure freedom after surgery more frequently [48]. Therefore we suggest from our study that a loss of whole GMV in epileptic Beagles is associated with a poorer outcome than higher whole grey matter volumes. VBM could therefore be further evaluated as a biomarker for treatment outcome.

\section{Conclusion}

In conclusion, in the current study the hypothesis was confirmed that epileptic Beagles have reduced GMV, especially in hippocampus and basal nuclei, as well as increased GMV in frontal regions of the brain. Additionally, further GMV differences between epileptic and healthy Beagles could be detected. All findings of volumetric differences between epileptic and healthy Beagles are in agreement with several findings in epileptic humans that emphasizes that the dog is a useful large animal model for humans [91-93]. Found reduction of grey matter volume in epileptic Beagles may represent 
the cause or consequence of epileptic seizures as well as be responsible for behavior changes of epileptic dogs, while an increased volume of grey matter can denote tissue regeneration or protective mechanisms. In this pilot study, it could be shown that VBM analysis represents an excellent method to detect GMV differences of the brain between groups of healthy and epileptic dogs, when MRI images of only one breed are compared. VBM cannot be recommended for evaluation of individual patients. Nevertheless, this technique has to be further developed in order to apply it broadly in veterinary medicine research.

\section{Abbreviations}

AED: Antiepileptic drugs; CSF: Cerebrospinal fluid; FWE: Family-wise error correction; FLE: Frontal lobe epilepsy; GM: Grey matter; GMV: Grey matter volume; GTCS: Generalized tonic-clonic seizures; IE: Idiopathic epilepsy; IGE: Idiopathic generalized epilepsy; JAE: Juvenile absence epilepsy; JME: Juvenile myoclonic epilepsy; MR: Magnetic resonance; MRI: Magnetic resonance imaging; SE: Structural epilepsy; T1W: T1-weighted; TFE: Turbo field echo; TLE: Temporal lobe epilepsy; TPM(s): Tissue probability map(s); VBM: Voxel-based morphometry; WBV: Whole brain volume; WM: White matter; WMV: white matter volume
\end{abstract}

\section{Acknowledgements}

The authors would like to thank Prof. Dr. Matthias Koepp from the Department of Clinical and Experimental Epilepsy, Institute of Neurology, London, for the helpful advice during the study; Dr. Peter Dziallas, Department of Small Animal Medicine and Surgery, University of Veterinary Medicine, Hannover, for the production of MR images and Prof. Dr. Hagen Gasse, Institute of Anatomy, University of Veterinary Medicine Hannover, for help in identifying anatomical structures.

\section{Funding}

Not applicable.

\section{Availability of data and materials}

The datasets used and analyzed during the current study are available from the corresponding author on reasonable request.

\section{Authors' contributions}

AT, ML and $W L$ conceived and designed the study, DK worked on methodology, LF and ML carried out methodology, LF and AT analyzed the data; LF wrote the manuscript and all authors read, contributed to and approved the final manuscript.

\section{Ethics approval and consent to participate}

MR images of control Beagles were already available from another animal experiment (Bach, JP et al..., 2013) that were approved by the Animal Welfare Officer of the University of Veterinary Medicine Hannover and the Lower Saxony State Office for Consumer Protection and Food Safety, Oldenburg, Germany (TV-No. 33.9-42,502-05-12A223). MR images of epileptic Beagles were made on request of their owners.

\section{Consent for publication}

Not applicable.

\section{Competing interests}

This publication is part of a thesis. The authors hereby declare that they have no competing interests that might have influenced the content of the manuscript.

\section{Publisher's Note}

Springer Nature remains neutral with regard to jurisdictional claims in published maps and institutional affiliations.

\section{Author details}

'Department of Small Animal Medicine and Surgery, University of Veterinary Medicine, Hannover, Germany. ²Department of General Radiology and Medical Physics, University of Veterinary Medicine, Hannover, Germany. ${ }^{3}$ Department of Pharmacology, Toxicology and Pharmacy, University of Veterinary Medicine, Hannover, Germany.

Received: 18 November 2017 Accepted: 14 February 2018

Published online: 20 February 2018

\section{References}

1. Berendt M, Farquhar RG, Mandigers PJ, Pakozdy A, Bhatti SF, De Risio L, Fischer A, Long S, Matiasek K, Munana K, et al. International veterinary epilepsy task force consensus report on epilepsy definition, classification and terminology in companion animals. BMC Vet Res. 2015;11:182.

2. Monteiro R, Adams V, Keys D, Platt SR. Canine idiopathic epilepsy: prevalence, risk factors and outcome associated with cluster seizures and status epilepticus. J Small Anim Pract. 2012;53(9):526-30.

3. Rusbridge C: Canina idiopathic epilepsy.Pdf. In Practice Focus 2014, 36:17-23.

4. Hülsmeyer V-I, Fischer A, Mandigers PJJ, DeRisio L, Berendt M, Rusbridge C, Bhatti SFM, Pakozdy A, Patterson EE, Platt S, et al. International veterinary epilepsy task Force's current understanding of idiopathic epilepsy of genetic or suspected genetic origin in purebred dogs. BMC Vet Res. 2015;11(1):175.

5. Weissl J, Hulsmeyer V, Brauer C, Tipold A, Koskinen LL, Kyostila K. Disease progression and treatment response of idiopathic epilepsy in Australian shepherd dogs. Vet Intern Med. 2012;26

6. Koestner A, Rehfeld CE. Idiopathic epilepsy in a beagle colony. ANL-7535. Anl. 1968:178-9.

7. Seppala EH, Koskinen LL, Gullov CH, Jokinen P, Karlskov-Mortensen P, Bergamasco L. Identification of a novel idiopathic epilepsy locus in Belgian shepherd dogs. PLoS One. 2012;7

8. Hulsmeyer V, Zimmermann R, Brauer C, Sauter-Louis C, Fischer A. Epilepsy in border collies: clinical manifestation, outcome, and mode of inheritance. Vet Intern Med. 2010;24

9. Patterson EE, Armstrong PJ, O'Brien DP, Roberts MC, Johnson GS, Mickelson JR. Clinical description and mode of inheritance of idiopathic epilepsy in English springer spaniels. J Am Vet Med Assoc. 2005;226(1):54-8.

10. Srenk P, Jaggy A, Gaillard C, Busato A, Horin P. Genetic basis of idiopathic epilepsy in the golden retriever. Tierarztliche Praxis. 1994;22(6):574-8.

11. Hall SJ, Wallace ME. Canine epilepsy: a genetic counselling programme for keeshonds. The Veterinary record. 1996;138(15):358-60.

12. Jaggy A, Faissler D, Gaillard C, Srenk P, Graber H. Genetic aspects of idiopathic epilepsy in Labrador retrievers. J Small Anim Pract. 1998;39(6): 275-80.

13. Jokinen TS, Metsahonkala L, Bergamasco L, Viitmaa R, Syrja P, Lohi H. Benign familial juvenile epilepsy in Lagotto Romagnolo dogs. J Vet Intern Med. 2007;21

14. Seppala EH, Jokinen TS, Fukata M, Fukata Y, Webster MT, Karlsson EK. LGI2 truncation causes a remitting focal epilepsy in dogs. PLoS Genet. 2011;7: e1002194.

15. Patterson EE, Mickelson JR, Da Y, Roberts MC, McVey AS, O'Brien DP. Clinical characteristics and inheritance of idiopathic epilepsy in Vizslas. J Vet Intern Med. 2003:17

16. De Lahunta A, Glass E. Veterinary neuroanatomy and clinical neurology. 3rd ed. Elsevier, Saunders: St. Louis, Missouri; 2009.

17. De Risio L, Bhatti S, Munana K, Penderis J, Stein V, Tipold A, Berendt M, Farqhuar R, Fischer A, Long S, et al. International veterinary epilepsy task force consensus proposal: diagnostic approach to epilepsy in dogs. BMC Vet Res. 2015;11:148.

18. Rusbridge C, Long S, Jovanovik J, Milne M, Berendt M, Bhatti SFM, De Risio L, Farqhuar RG, Fischer A, Matiasek K, et al. International veterinary epilepsy task force recommendations for a veterinary epilepsy-specific MRI protocol. BMC Vet Res. 2015;11(1):194.

19. Keller SS, Roberts N. Voxel-based morphometry of temporal lobe epilepsy: an introduction and review of the literature. Epilepsia. 2008;49(5):741-57.

20. Ashburner J, Friston KJ. Voxel-based morphometry-the methods. Neurolmage. 2000;11(6 Pt 1):805-21.

21. Whitwell JL. Voxel-based morphometry: an automated technique for assessing structural changes in the brain. J Neurosci. 2009;29(31):9661-4. 
22. Milne ME, Steward C, Firestone SM, Long SN, O'Brien TJ, Moffat BA. Development of representative magnetic resonance imaging-based atlases of the canine brain and evaluation of three methods for atlas-based segmentation. AJVR No. 2016;77(4):395-403.

23. Wrzosek M, Podgórski P, Drobot P, Bodys W, Nicpoń J: The MR Volumetric Brain Assessment in Canine Epileptic Patients - A Pilot Study [abstract]. Congress Proceedings of the 29th Symposium of the ESVN-ECVN Edinburgh, United Kingdom 16th \& 17th September 20162016.

24. Kuwabara T, Hasegawa D, Kobayashi M, Fujita M, Orima H. Clinical magnetic resonance Volumetry of the hippocampus in 58 epileptic dogs*. Veterinary Radiology \& Ultrasound. 2010;51(5):485-90.

25. Milne M, Moffat, B., O'Brien, T., Long, S.: Atlas based segmentation identifies hippocampal atrophy in epileptic dogs. Abstracts of the 17th International Veterinary Radiology Association Meeting, Perth, Australia 16th to 21st August 20152015.

26. Bin G, Wang T, Zeng H, He X, Li F, Zhang J, Huang B. Patterns of gray matter abnormalities in idiopathic generalized epilepsy: a meta-analysis of voxel-based morphology studies. PLoS One. 2017;12(1):e0169076.

27. Bach JP, Lupke M, Dziallas P, Wefstaedt P, Uppenkamp S, Seifert H, Nolte I. Functional magnetic resonance imaging of the ascending stages of the auditory system in dogs. BMC Vet Res. 2013;9:210.

28. FAST - Tissue-Type Segmentation and Bias Field Correction [https://www. fmrib.ox.ac.uk/datasets/techrep/tr04ss2/tr04ss2/node11.html].

29. Tapp PD, Head K, Head E, Milgram NW, Muggenburg BA, Su MY. Application of an automated voxel-based morphometry technique to assess regional gray and white matter brain atrophy in a canine model of aging. Neurolmage. 2006;29(1):234-44.

30. Manual Computational Anatomy Toolbox - CAT12 [http://www.neuro.unijena.de/cat/].

31. VBM Tutorial [http://www.fil.ion.ucl.ac.uk/ john/misc/].

32. Uemura EE. Fundamentals of canine neuroanatomy and neurophysiology. Ames, lowa: John Wiley \& Sons, Inc.; 2015

33. Stoffel MH. Funktionelle Neuroanatomie für die Tiermedizin. Enke Verlag: Stuttgard; 2010.

34. Palazzi $X$. The beagle brain in stereotaxic coordinates. New York, NY: Springer; 2011.

35. Schulze S, Schmidt M, Nitzsche B: Canine brain Project: Morphometrie zerebraler magnetresonanztomographischer Datensätze verschiedener Hunderassen. Proceedings des 62 Jahreskongresses der DGK-DVG, 27-30 Oktober 2016, Berlin 2016.

36. Datta R, Lee J, Duda J, Avants BB, Vite CH, Tseng B, Gee JC, Aguirre $\mathrm{GD}$, Aguirre GK. A digital atlas of the dog brain. PLoS One. 2012;7(12):e52140.

37. Ogata N, Gillis TE, Liu X, Cunningham SM, Lowen SB, Adams BL, SutherlandSmith J, Mintzopoulos D, Janes AC, Dodman NH, et al. Brain structural abnormalities in Doberman pinschers with canine compulsive disorder. Prog Neuro-Psychopharmacol Biol Psychiatry. 2013;45:1-6.

38. Abell F, Krams M, Ashburner J, Passingham R, Friston K, Frackowiak R, Happé F, Frith C, Frith U. The neuroanatomy of autism: a voxel-based whole brain analysis of structural scans. Neuroreport. 1999;10(8):1647-51.

39. Douaud G, Smith S, Jenkinson M, Behrens T, Johansen-Berg H, Vickers J, James S, Voets N, Watkins K, Matthews PM, et al. Anatomically related grey and white matter abnormalities in adolescent-onset schizophrenia. Brain. 2007;130:2375-86.

40. Ferreira LK, Diniz BS, Forlenza OV, Busatto GF, Zanetti MV. Neurostructural predictors of Alzheimer's disease: a meta-analysis of VBM studies. Neurobiol Aging. 2011;32(10):1733-41

41. Prinster A, Quarantelli M, Orefice G, Lanzillo R, Brunetti A, Mollica C, Salvatore E, Morra VB, Coppola G, Vacca G, et al. Grey matter loss in relapsing-remitting multiple sclerosis: a voxel-based morphometry study. Neurolmage. 2006;29(3):859-67.

42. Yasuda CL, Betting LE, Cendes F. Voxel-based morphometry and epilepsy. Expert Rev Neurother. 2010;10(6):975-84.

43. Tapp D, Head K, Head E, Cotman CW, Milgram NW, Nalcioglu O, Su M-Y. Use of voxel-based morphometry techniques to assess the benefits of an antioxidant diet in preventing cortical atrophy in a dog model of brain aging. Proc Intl Soc Mag Reson Med. 2005;13:1024.

44. Milne M, Moffat B, O'Brien T, Long S. Atlas based segmentation identifies hippocampal atrophy in epileptic dogs. Veterinary Radiology and Ultrasound. 2016;57(2):207-22.
45. Bonilha L, Rorden C, Appenzeller S, Coan AC, Cendes F, Li LM. Gray matter atrophy associated with duration of temporal lobe epilepsy. Neurolmage. 2006;32(3):1070-9.

46. Bernasconi N, Duchesne S, Janke A, Lerch J, Collins DL, Bernasconi A. Whole-brain voxel-based statistical analysis of gray matter and white matter in temporal lobe epilepsy. Neurolmage. 2004;23(2):717-23.

47. Keller S, Wieshmann U, Mackay C, Denby C, Webb J, Roberts N. Voxel based morphometry of grey matter abnormalities in patients with medically intractable temporal lobe epilepsy: effects of side of seizure onset and epilepsy duration. J Neurol Neurosurg Psychiatry. 2002;73(6):648-55.

48. Doucet GE, He X, Sperling M, Sharan A, Tracy Jl. Frontal gray matter abnormalities predict seizure outcome in refractory temporal lobe epilepsy patients. Neurolmage Clinical. 2015;9:458-66.

49. Mueller SG, Laxer KD, Cashdollar N, Buckley S, Paul C, Weiner MW. Voxelbased optimized morphometry (VBM) of gray and white matter in temporal lobe epilepsy (TLE) with and without mesial temporal sclerosis. Epilepsia. 2006:47(5):900-7.

50. Woermann FG, Free SL, Koepp MJ, Ashburner J, Duncan JS. Voxel-by-voxel comparison of automatically segmented cerebral gray matter a raterindependent comparison of structural MRI in patients with epilepsy.Pdf. Neurolmage. 1999;10:373-84.

51. de Araujo Filho GM, Jackowski AP, Lin K, Guaranha MS, Guilhoto LM, da Silva HH, Caboclo LO, Junior HC, Bressan RA, Yacubian EM. Personality traits related to juvenile myoclonic epilepsy: MRI reveals prefrontal abnormalities through a voxel-based morphometry study. Epilepsy Behav. 2009;15(2):202-7.

52. Tondelli M, Vaudano AE, Ruggieri A, Meletti S. Cortical and subcortical brain alterations in juvenile absence epilepsy. Neurolmage Clinical. 2016;12:306-11.

53. Huang W, Lu G, Zhang Z, Zhong Y, Wang Z, Yuan C, Jiao Q, Qian Z, Tan Q, Chen $\mathrm{G}$, et al. Gray-matter volume reduction in the thalamus and frontal lobe in epileptic patients with generalized tonic-clonic seizures. J Neuroradiol. 2011;38(5):298-303.

54. Braakman HM, Vaessen MJ, Jansen JF, Debeij-van Hall MH, de Louw A, Hofman PA, Vles JS, Aldenkamp AP, Backes WH. Aetiology of cognitive impairment in children with frontal lobe epilepsy. Acta Neurol Scand. 2015;131(1):17-29.

55. Bonilha L, Rorden C, Castellano G, Pereira F, Rio PA, Cendes F, Li LM. Voxelbased morphometry reveals gray matter network atrophy in refractory medial temporal lobe epilepsy. Arch Neurol. 2004;61(9):1379-84.

56. Dupont S, Ottaviani M, Thivard L, Semah F, Samson Y, Baulac M. Temporal pole hypometabolism may be linked to a reduction of grey matter in temporal lobe epilepsy. Neuroreport. 2002;13(18):2537-41.

57. Lin K, Jackowski AP, Carrete H Jr, de Araujo Filho GM, Silva HH, Guaranha MS, Guilhoto LM, Bressan RA, Yacubian EM. Voxel-based morphometry evaluation of patients with photosensitive juvenile myoclonic epilepsy. Epilepsy Res. 2009;86(2-3):138-45.

58. Woermann FG, van Elst LT, Koepp MJ, Free SL, Thompson PJ, Trimble MR, Duncan JS. Reduction of frontal neocortical grey matter associated with affective aggression in patients with temporal lobe epilepsy: an objective voxel by voxel analysis of automatically segmented MRI. J Neurol Neurosurg Psychiatry. 2000;68(2):162-9.

59. Lieb JP, Dasheiff RM, Engel J Jr. Role of the frontal lobes in the propagation of mesial temporal lobe seizures. Epilepsia. 1991;32(6):822-37.

60. Adam C, Saint-Hilaire JM, Richer F. Temporal and spatial characteristics of intracerebral seizure propagation: predictive value in surgery for temporal lobe epilepsy. Epilepsia. 1994;35(5):1065-72.

61. Morita T, Shimada A, Takeuchi T, Hikasa Y, Sawada M, Ohiwa S. Cliniconeuropathologic findings of familial frontal lobe epilepsy in Shetland sheepdogs. Can J Vet Res. 2002;66

62. Michelucci R, Poza JJ, Sofia V, de Feo MR, Binelli S, Bisulli F, Scudellaro E, Simionati B, Zimbello R, D'Orsi G, et al. Autosomal dominant lateral temporal epilepsy: clinical spectrum, new epitempin mutations, and genetic heterogeneity in seven European families. Epilepsia. 2003;44(10):1289-97.

63. Wessmann A, Volk HA, Packer RM, Ortega M, Anderson TJ. Quality-of-life aspects in idiopathic epilepsy in dogs. The Veterinary record. 2016;179(9):229.

64. Betting LE, Mory SB, Li LM, Lopes-Cendes I, Guerreiro MM, Guerreiro CA, Cendes F. Voxel-based morphometry in patients with idiopathic generalized epilepsies. Neurolmage. 2006;32(2):498-502.

65. Kim JH, Lee JK, Koh SB, Lee SA, Lee JM, Kim SI, Kang JK. Regional grey matter abnormalities in juvenile myoclonic epilepsy: a voxel-based morphometry study. Neurolmage. 2007;37 
66. Meencke HJ. Neuron density in the molecular layer of the frontal cortex in primary generalized epilepsy. Epilepsia. 1985;26(5):450-4.

67. Meencke HJ, Janz D. Neuropathological findings in primary generalized epilepsy: a study of eight cases. Epilepsia. 1984;25(1):8-21.

68. Meiners LC, van Gils A, Jansen GH, de Kort G, Witkamp TD, Ramos LM, Valk J, Debets RM, van Huffelen AC, van Veelen CW, et al. Temporal lobe epilepsy: the various MR appearances of histologically proven mesial temporal sclerosis. AJNR Am J Neuroradiol. 1994;15(8):1547-55.

69. Doucet GE, He X, Sperling M, Sharan A, Tracy Jl. Gray matter abnormalities in temporal lobe epilepsy: relationships with resting-state functional connectivity and episodic memory performance. PLoS One. 2016;11(5):e0154660.

70. O'Muircheartaigh J, Vollmar C, Barker GJ, Kumari V, Symms MR, Thompson P, Duncan JS, Koepp MJ, Richardson MP. Focal structural changes and cognitive dysfunction in juvenile myoclonic epilepsy. Neurology. 2011;76(1):34-40.

71. Braga AM, Fujisao EK, Verdade RC, Paschoalato RP, Paschoalato RP Yamashita S, Betting LE. Investigation of the cingulate cortex in idiopathic generalized epilepsy. Epilepsia. 2015;56(11):1803-11.

72. Dewey CW, da Costa RC. Practical guide to canine and feline neurology. Ames, lowa: John Wiley \& Sons, Inc.; 2016.

73. Peng S-J, Harnod T, Tsai J-Z, Ker M-D, Chiou J-C, Chiueh H, Wu C-Y, Hsin Y-L. Evaluation of subcortical grey matter abnormalities in patients with MRInegative cortical epilepsy determined through structural and tensor magnetic resonance imaging. BMC Neurol. 2014;14(1):104.

74. Seeck M, Dreifuss S, Lantz G, Jallon P, Foletti G, Despland P-A, Delavelle J, Lazeyras F. Subcortical nuclei Volumetry in idiopathic generalized epilepsy. Pdf. Epilepsia. 2005;46:1642-5.

75. Du H, Zhang Y, Xie B, Wu N, Wu G, Wang J, Jiang T, Feng H. Regional atrophy of the basal ganglia and thalamus in idiopathic generalized epilepsy. J Magn Reson Imaging. 2011;33

76. Pulsipher DT, Seidenberg M, Morton JJ, Geary E, Parrish J, Hermann B. MRI volume loss of subcortical structures in unilateral temporal lobe epilepsy. Epilepsy Behav. 2007;11

77. Duzel E, Schiltz K, Solbach T, Peschel T, Baldeweg T, Kaufmann J, Szentkuti A, Heinze HJ. Hippocampal atrophy in temporal lobe epilepsy is correlated with limbic systems atrophy. J Neurol. 2006;253(3):294-300.

78. Nakayama $Y$, Masuda H, Shirozu H, Ito Y, Higashijima T, Kitaura H, Fujii Y, Kakita A, Fukuda M. Features of amygdala in patients with mesial temporal lobe epilepsy and hippocampal sclerosis: an MRI volumetric and histopathological study. Epilepsy Res. 2017;135:50-5.

79. Engel J Jr. Mesial temporal lobe epilepsy: what have we learned? Neuroscientist. 2001;7(4):340-52.

80. Blades Golubovic S, Rossmeisl JH Jr. Status epilepticus in dogs and cats, part 1: etiopathogenesis, epidemiology, and diagnosis. J Vet Emerg Crit Care (San Antonio). 2017;27(3):278-87.

81. Tapp PD, Siwak CT, Gao FQ, Chiou JY, Black SE, Head E, Muggenburg BA, Cotman CW, Milgram NW, Su MY. Frontal lobe volume, function, and beta-amyloid pathology in a canine model of aging. J Neurosci. 2004;24(38):8205-13.

82. Montgomery DL, Lee AC. Brain damage in the epileptic beagle dog Vet Pathol. 1983;20(2):160-9.

83. Nevander G, Ingvar M, Auer R, Siesjo BK. Status epilepticus in welloxygenated rats causes neuronal necrosis. Ann Neurol. 1985;18(3):281-90.

84. Bouilleret V, Nehlig A, Marescaux C, Namer IJ. Magnetic resonance imaging follow-up of progressive hippocampal changes in a mouse model of mesial temporal lobe epilepsy. Epilepsia. 2000;41(6):642-50.

85. Keller SS, Wilke M, Wieshmann UC, Sluming VA, Roberts N. Comparison of standard and optimized voxel-based morphometry for analysis of brain changes associated with temporal lobe epilepsy. Neurolmage. 2004;23(3):860-8.

86. Blumenfeld H, Varghese Gl, Purcaro MJ, Motelow JE, Enev M, McNally KA, Levin AR, Hirsch LJ, Tikofsky R, Zubal IG, et al. Cortical and subcortical networks in human secondarily generalized tonic-clonic seizures. Brain. 2009;132

87. Marcián V, Filip P, Bareš M, Brázdil M. Cerebellar dysfunction and ataxia in patients with epilepsy: coincidence, consequence, or cause? Tremor and Other Hyperkinetic Movements. 2016;6:376.

88. Crooks R, Mitchell T, Thom M. Patterns of cerebellar atrophy in patients with chronic epilepsy: a quantitative neuropathological study. Epilepsy Res. 2000;41(1):63-73.
89. Salcman M, Defendini R, Correll J, Gilman S. Neuropathological changes in cerebellar biopsies of epileptic patients. Ann Neurol. 1978;3(1):10-9.

90. Savic I, Altshuler L, Passaro E, Baxter L, Engel J Jr. Localized cerebellar hypometabolism in patients with complex partial seizures. Epilepsia. 1996:37(8):781-7.

91. Potschka H, Fischer A, von Ruden EL, Hulsmeyer V, Baumgartner W. Canine epilepsy as a translational model? Epilepsia. 2013;54(4):571-9.

92. Löscher W. Animal models of intractable epilepsy. Prog Neurobiol. 1997:53(2):239-58.

93. Löscher W, Schwartz-Porsche D, Frey HH, Schmidt D. Evaluation of epileptic dogs as an animal model of human epilepsy. Arzneimittelforschung. 1985;35(1):82-7.

\section{Submit your next manuscript to BioMed Central and we will help you at every step:}

- We accept pre-submission inquiries

- Our selector tool helps you to find the most relevant journal

- We provide round the clock customer support

- Convenient online submission

- Thorough peer review

- Inclusion in PubMed and all major indexing services

- Maximum visibility for your research

Submit your manuscript at www.biomedcentral.com/submit 